\title{
More Rare Males in Ostrinia: Response of Asian Corn Borer Moths to the Sex Pheromone of the European Corn Borer
}

\author{
Charles E. Linn Jr. • Callie J. Musto • \\ Wendell L. Roelofs
}

Received: 21 June 2006 /Revised: 5 September 2006 / Accepted: 12 September 2006 /

Published online: 5 December 2006

(C) Springer Science + Business Media, Inc. 2006

\begin{abstract}
A previous flight tunnel study showed that 3-5\% of European corn borer (ECB) moths, Ostrinia nubilalis (Z/E11-14:OAc), could fly upwind and make contact with sources releasing the sex pheromone of the related Asian corn borer (ACB), Ostrinia furnacalis (2:1 Z/E12-14:OAc). In this study, we show that rare males (3-4\%) are also present in South Korean ACB that respond to the sex pheromone blends of the ECB UZ (97:3 Z/E11-14: $\mathrm{OAc})$ and $\mathrm{BE}$ (1:99 Z/E11-14:OAc) pheromone races. We also show that the upwind flight response of a significant proportion of male ACB was antagonized by the addition of $1 \%$ 29-14:OAc to the ACB blend, a compound that also antagonizes the upwind flight of ECB males. Male ACB flight behavior was not, however, affected by adding either of the ECB blends to the ACB blend, or by the addition of 50\% 14:OAc, a compound identified from female pheromone glands of ACB and a number of other Ostrinia species. Additional flight tunnel tests with $\mathrm{ACB}$ to study the comparative aspects of $\mathrm{ECB}$ and $\mathrm{ACB}$ pheromone response specificity showed that male ACB exhibited maximal levels of upwind flight and source contact with doses of pheromone (30 and $100 \mu \mathrm{g}$ on rubber septum sources) that also elicited maximal levels in the two ECB pheromone races. The maximal level of source contact for ACB (66\%) was lower than observed with the UZ race of ECB to its pheromone blend ( $>95 \%)$, but comparable to those for the BE race of ECB (65-70\%). Male ACB also flew upwind in high proportions to a broader range of ratios of Z/E12-14:OAc (80:20 to 20:80) than was previously observed for either of the ECB races.
\end{abstract}

Keywords Asian corn borer - Behavioral antagonist - European corn borer · Flight tunnel

C. E. Linn Jr. $(\bowtie) \cdot$ C. J. Musto $•$ W. L. Roelofs

Department of Entomology, Barton Lab, New York State Agricultural Experiment Station,

Cornell University, 630 W. North Street, Geneva, NY 14456, USA

e-mail: CEL1@cornell.edu 
Ostrinia furnacalis · Ostrinia nubilalis $\cdot$ Pheromone evolution $\cdot$ Response specificity Sex pheromone

\section{Introduction}

The genus Ostrinia (Lepidoptera: Crambidae) is composed of 20 species, including two of agricultural importance, the European corn borer (ECB), Ostrinia nubilalis (Hübner), and the Asian corn borer (ACB), Ostrinia furnacalis. The ECB has been studied more extensively and exists in Europe and North America as two distinct pheromone races (Kochansky et al., 1975; Roelofs et al., 1985). Most populations ( $Z$ race) use a 97:3 blend of $(Z)-11$ - and (E)-11-tetradecenyl acetates (Z11/E11-14:OAc), but occur in some areas sympatrically with an $E$ race that uses the opposite 1:99 Z/E blend. In New York state, three different races of ECB, based on the sex pheromone blend and the number of generations (voltinism), have been described: bivoltine $\mathrm{Z}$ (designated BZ), univoltine $\mathrm{Z}$ (UZ), and bivoltine E (BE) (Roelofs et al., 1985). Studies of the genetic basis of the ECB pheromone system have shown that there are three genes responsible for the differences (Roelofs et al., 1987; Löfstedt et al., 1989; Cossé et al., 1995).

The ACB, which occupies the ecological niche in Asia equivalent to that occupied by ECB in Europe (Ishikawa et al., 1999a), uses mixtures of (Z)-12- and (E)-12-tetradecenyl acetates (Z12/E12-14:OAc) that vary from 1:1 in the Philippines, mainland China, Taiwan, and some parts of Japan (Klun et al., 1980; Cheng et al., 1981; Du et al., 1986; Kou et al., 1992) to 2:1 in the southernmost part of Japan (Ando et al., 1980; Huang et al., 1998) and South Korea (Boo and Park, 1998).

In studies on the desaturase enzymes involved in controlling pheromone biosynthesis, Roelofs et al. (2002) discovered that the female pheromone gland of the ECB contains the expected desaturase gene that makes $\Delta 11-14$ :Acid for production of its pheromone components, but the gland also contains the gene for a $\Delta 14-16$ :Acid desaturase used by the ACB. The ACB pheromone gland likewise contains the same two desaturase genes, but only expresses the $\Delta 14$ desaturase, which it uses for its pheromone components. Roelofs et al. (2002) proposed that a saltational event occurred in an ancestral Ostrinia moth population and gave rise to a new pheromone and new species, the ACB. A possible pseudogene that had been unexpressed for millions of years in the genome of Ostrinia became functional and generated pheromone precursor fatty acids that differed in double-bond position and chain length. The resulting pheromone acetates then possessed a double bond in the $\Delta 12$ position instead of the $\Delta 11$ position. The desaturase studies raised the possibility that a new pheromone communication system can arise as a result of changes in regulatory pathways controlling gene expression. However, for the new pheromone to be effective in mate location it is necessary for some males to be able to detect and respond to the new blend. Subsequent flight tunnel studies showed that some males (3-5\%) in the $\mathrm{UZ}$ and BE $O$. nubilalis races did, in fact, respond to both species' pheromone blends (Linn et al., 2003).

In this study, we report the results of flight tunnel experiments showing that there also are rare ACB males from a population originating in South Korea that respond to one or both of the ECB blends. We show further that the upwind flight of ACB males to their pheromone is antagonized by the addition of Z9-14:OAc, as it occurs with ECB, but not by the addition of the ECB blends to the ACB mixture. We also provide support for the claim that tetradecyl acetate (14:OAc), although present in the pheromone glands of many Ostrinia species, including ACB, does not enhance or disrupt levels of upwind flight. 


\section{Methods and Materials}

Insects

Colonies of the $\mathrm{UZ}$ and BE races of $O$. nubilalis from New York state were maintained in separate walk-in environmental chambers. Mating and larval rearing conditions were as described in Roelofs et al. (1987) at a constant temperature of $25^{\circ} \mathrm{C}$ and $16: 8 \mathrm{~L} / \mathrm{D}$ photoperiod. Pupae were sexed and the male pupae were placed on a layer of vermiculite in plastic and screen emergence cages inside a walk-in environmental chamber $\left(25^{\circ} \mathrm{C}, 16: 8 \mathrm{~L} / \mathrm{D}\right.$ photoperiod). Cages of adults were separated daily so that individuals of known age could be used for flight tunnel tests.

The ACB were obtained from Jin Kyo Jung, National Institute of Crop Sciences, South Korea, and maintained on the same diet and environmental conditions in the lab in New York as were the ECB populations.

\section{Chemicals}

Z11-14:OAc, E11-14:OAc, Z12-14:OAc, E12-14:OAc, Z9-14:OAc, and 14:OAc were obtained from the Pherobank (http://www.pherobank.nl), and mixtures were prepared in high-performance liquid chromatography grade hexane and applied to red rubber septa (Thomas Scientific, Swedesboro, NJ, USA; Glover et al., 1989; Linn et al., 1997).

\section{Flight Tunnel}

Adults were tested in the sustained flight tunnel during their second to third night after ecolosion under standard conditions for O. nubilalis (Glover et al., 1989; Linn et al., 1997): $20-21{ }^{\circ} \mathrm{C}, 60-65 \% \mathrm{RH}, 0.50 \mathrm{~m} / \mathrm{sec}$ air flow, and illumination of 11 lux of red light at the tunnel floor during the third to sixth hour of scotophase. Adults were taken to the room housing the flight tunnel $1 \mathrm{hr}$ before the start of the eighth hour of scotophase and placed individually in screen release cages so they could acclimate to the flight tunnel room environment. The temperature during the 1-hr period of acclimation, in photophase, was $25^{\circ} \mathrm{C}$, then, when lights off occurred the temperature dropped to $20-21^{\circ} \mathrm{C}$. Adult moths were tested individually, and a positive response was counted if the male exhibited upwind flight in the odor plume and made contact with the rubber septum source.

Six experiments were conducted, each designed to address a different question about the comparative response specificity of ACB and ECB males. In some cases, individual males were tested to more than one treatment during each test period. After recapture, males were removed from the flight tunnel and allowed a minimum period of $15 \mathrm{~min}$ before retesting to a different blend. Previous experiments have demonstrated that ECB can be tested up to four times in a daily testing period without adverse affects on performance (Cossé et al., 1995). Preliminary experiments indicated that ACB could also be tested three times during a single testing period with no adverse affects on levels of upwind flight.

\section{Experiment 1: Response of ACB Males to a Dose Series of Pheromone}

ACB males were tested to a dose series of the 2:1 Z/E12-14:OAc blend: 1, 3, 30, 100, and $300 \mu \mathrm{g} / \mathrm{septum}$. During each testing period, a minimum of $10 \mathrm{ACB}$ males were tested to at 
least two of the five doses, with each dose tested a minimum of four different testing periods (days).

\section{Experiment 2: Response of ACB Males to Different Blends of Z/E12-14:OAc}

ACB males were tested to $30-\mu \mathrm{g}$ doses of the following Z/E12-14:OAc blends: 4:1, 2:1, $1: 1,1: 2$, and 1:4. During each day of testing, a minimum of 10 males were tested to three of the blends, with treatment blends rotated over subsequent days to achieve approximately the same number of males tested to each blend.

Experiment 3: Are There Rare ACB Males that Respond to the ECB Blends?

Two protocols were used for this experiment. In the first, ACB males and UZ race ECB males were tested to a 30- $\mu$ g dose of the 2:1 Z/E12-14:OAc blend (ACB blend) and to a 30- $\mu$ g dose of the 97:3 Z/E11-14:OAc UZ ECB blend (UZ blend). Males of each species were tested during each daily test period, beginning with the ACB blend for UZ males and the UZ blend for ACB males. In the second protocol, ACB males were tested to 30- $\mathrm{gg}$ doses of the UZ blend, 1:99 Z/E11-14:OAc BE ECB blend (BE blend), and the ACB blend. Each male was tested sequentially to the three blends in the same testing period, beginning with either of the ECB blends and ending with the ACB blend.

Experiment 4: Effect of Z9-14:OAc on Male ACB Response to the ACB Pheromone Blend

ACB males were tested to a $30-\mu$ g dose of the ACB blend alone, or with 1\% Z9-14:OAc added to the mixture. The $1 \%$ value was chosen based on previous studies (Glover et al., 1989). For comparison, male ECB of the BE race were also tested on each day to a $30-\mu \mathrm{g}$ dose of the BE blend alone, or with 1\% Z9-14:OAc added to the mixture.

Experiment 5: Response of ACB Males to the Combined ACB/ECB Blends

ACB males were tested to a $30-\mu \mathrm{g}$ dose of the ACB blend alone or with a $30-\mu \mathrm{g}$ dose of the $\mathrm{UZ}$ or BE blends added.

Experiment 6: Response of ACB Males to the ACB Blend with 14:OAc Added

Previous studies with ACB have shown that 14:OAc is present in female pheromone glands, but has never been shown to enhance trap capture of moths (Boo and Park, 1998). In our experiment, ACB males were tested to a $30-\mu \mathrm{g}$ dose of the ACB blend alone, or with $50 \%$ of the compound $14:$ OAc added. The $50 \%$ value was relative to the amount of Z12-14:OAc in the blend and was selected based on the study by Boo and Park (1998) showing that female glands from 2- to 3-d-old females contained a mean ratio of 0.74:1:1.36 for 14:OAc, E12-14:OAc, and Z12-14:OAc, respectively.

\section{Statistical Analysis}

For each experiment the number of moths in each treatment that exhibited upwind flight to the source was combined for all testing periods and converted to a percent for graphical display. 
Chi-square analysis or post hoc pairwise comparisons using Fisher's exact tests were implemented according to the JMP statistical analysis program for Macintosh $P<0.05$ ).

\section{Results}

Experiment 1: Response of ACB Males to a Dose Series of Pheromone

The greatest percentage of source contacts by ACB males occurred with the 30- $\mu$ g dose of the ACB blend $(66 \%, N=40$; Fig. 1a). This value was not significantly different from the lower percentages with the $3-\mu \mathrm{g}(53 \%, N=53$; Fisher's exact test $P=0.273, d f=1)$ and $100-\mu \mathrm{g}(63 \%, N=44$; Fisher's exact test $P=0.825, d f=1)$ doses. However, with the $1-\mu \mathrm{g}$

Fig. 1 Percentage source contact by $\mathrm{ACB}$ males tested to a dose series $(1,3,30,100$, and $300 \mu \mathrm{g}$; $N=63,64,60,65$, and 62 , respectively) of the $2: 1 \mathrm{Z} / \mathrm{E} 12-14$ : OAc ACB pheromone (a). Data points with different letters are significantly different $(P<0.05)$ according to selected post hoc Fisher's exact tests (see text). For comparison, response values for ECB males (UZ males to $97: 3$ and $\mathrm{BE}$ males to $1: 99 \mathrm{Z} / E-11-14$ : $\mathrm{OAc}$ ) are presented in subpanel b, modified from Linn et al. (1997)
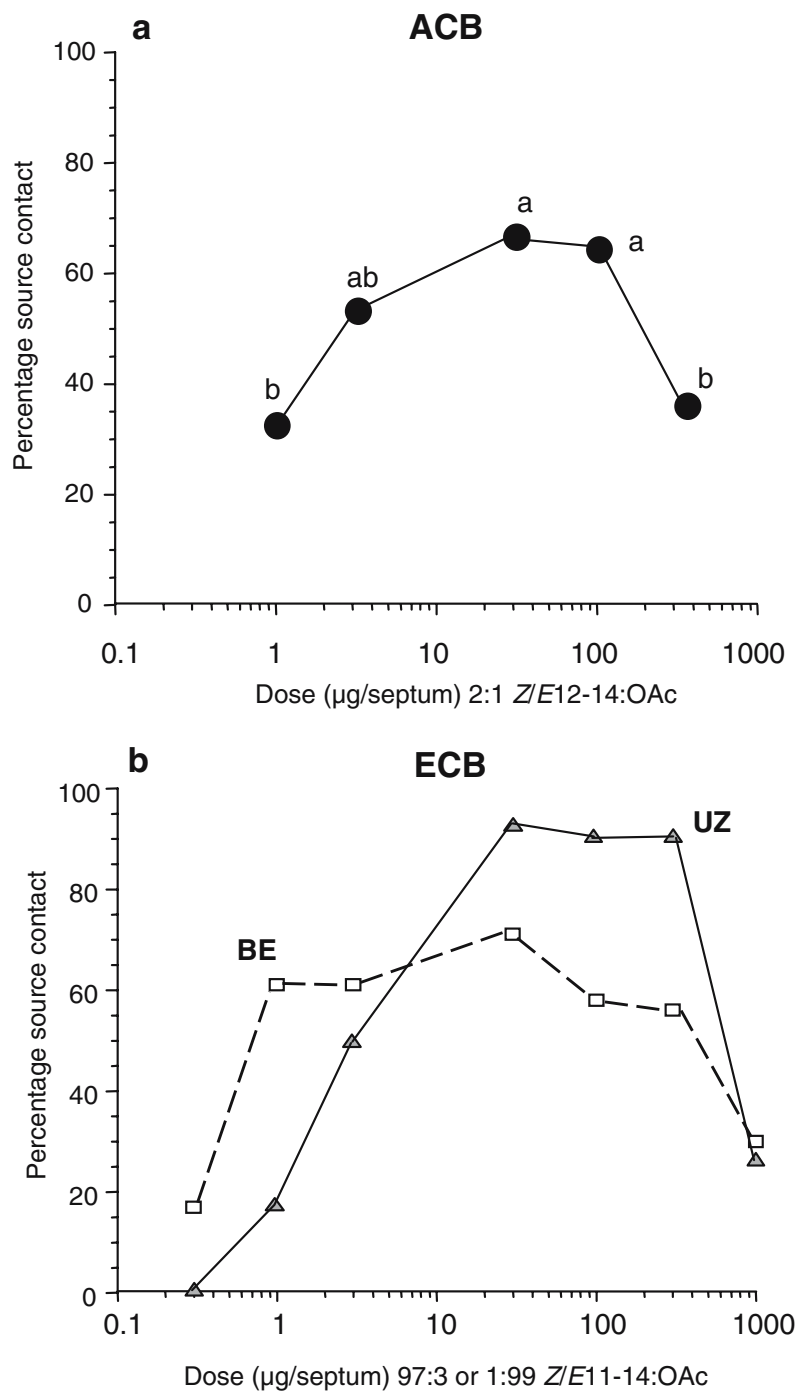
$(32 \%, N=43)$ and $300-\mu g(35 \%, N=42)$ doses, there were significant reductions in the percentages of source contacts compared with the 100- $\mu$ g dose (Fisher's exact test $P=$ $0.004, d f=1$ for $3 \mu \mathrm{g}$ vs. $100 \mu \mathrm{g}$ ). Based on these results, the $30-\mu \mathrm{g}$ dose of the ACB blend was used for the other experiments. Previous results with two populations of ECB also showed that $30-\mu \mathrm{g}$ of the $97: 3$ or 1:99 Z/E11-14:OAc blends elicited maximal response levels (Fig. 1b; modified from Linn et al., 1997; see also Glover et al., 1989). Maximal response levels were lower for the $\mathrm{BE}(72 \%, 30 \mu \mathrm{g})$ and $\mathrm{ACB}(66 \%, 30 \mu \mathrm{g})$ populations than for UZ males $(93 \%, 30 \mu \mathrm{g})$ (Fig. 1b).

\section{Experiment 2: Response of ACB Males to Different Blends of Z/E12-14:OAc}

There were no significant differences in the response levels of ACB males to the $30-\mu \mathrm{g}$ dose of five ratios of $Z / E 12-14$ :OAc (Fig. $2 \mathrm{a} ; \chi^{2}=5.296, P>0.05$ ). Interestingly, ACB

Fig. 2 Percentage source contact by ACB males tested to $30-\mu \mathrm{g}$ doses of five different ratios $(4: 1$, $2: 1,1: 1,1: 2$, and $1: 4 Z / E ; N=63$, $67,64,67$, and 62 , respectively) of the ACB pheromone (a). There were no significant differences in the response values $\left(\chi^{2}, P>0.05\right)$, and lines above and below data points indicate the range of values obtained over $6 \mathrm{~d}$ of testing. For comparison, response values for $\mathrm{UZ}$ and $\mathrm{BE}$ race, $\mathrm{ECB}$ males to different ratios of Z:E1114:OAc are presented in subpanel b, modified from Linn et al. (1997)
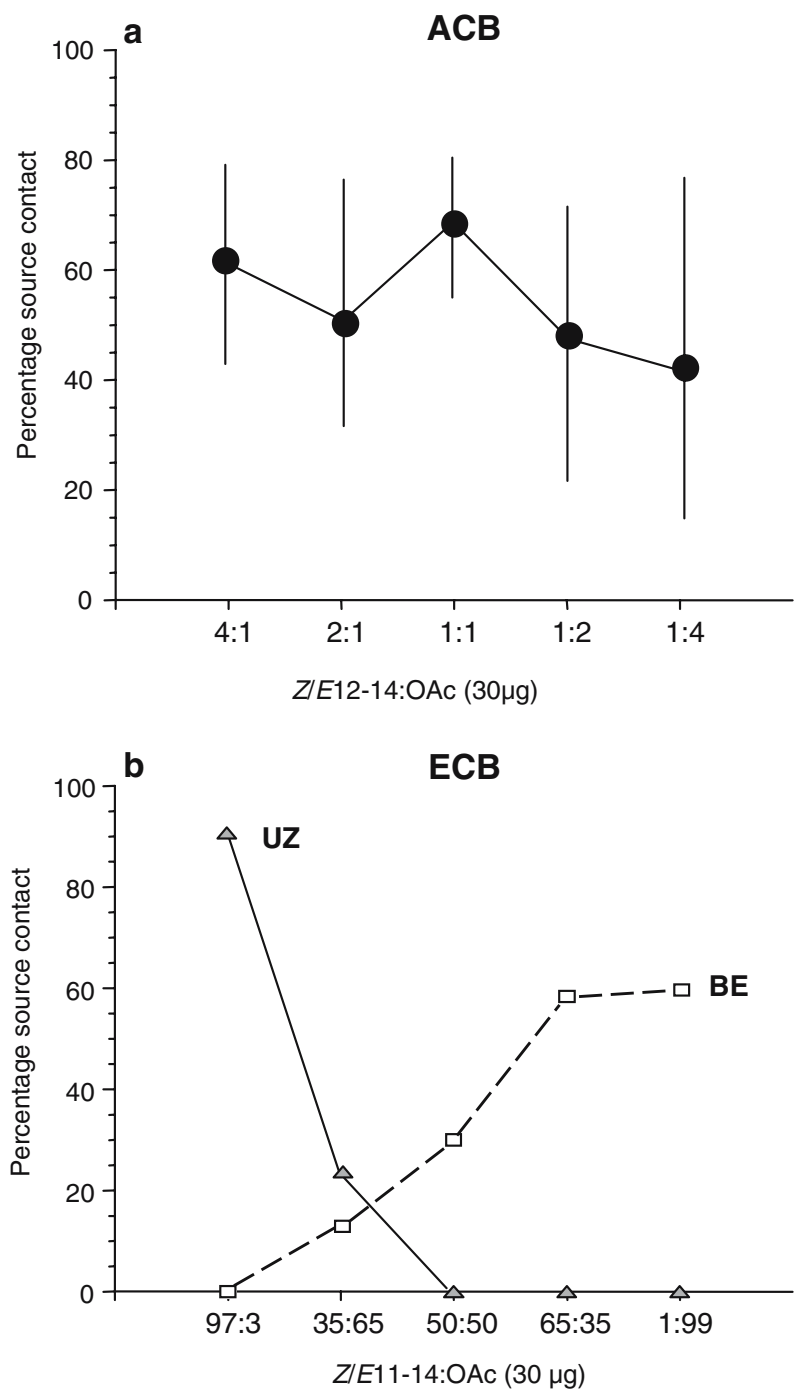
males flew upwind in the greatest percentage to the 1:1 Z/E12-14:OAc ratio (69\%), and not the 2:1 mixture that occurs in the glands (Boo and Park, 1998). The measurement of 50\% for source contacts with the 2:1 ratio in this experiment was the lowest measurement for that blend in all of the other experiments (generally in the $60-70 \%$ range, see below). For most of the ratios, there was considerable variability in the male preference for blend quality over the 6-d testing period (Fig. 2a).

Comparative data from Linn et al. (1997) for the upwind flight response of UZ and BE ECB males to a range of $Z / E 11-14$ :OAc ratios show that ACB males displayed a much lower preference for odor quality than did ECB males (Fig. 2b). UZ males displayed a high specificity for the 97:3 Z/E11-14:OAc blend that occurs in the glands, with $>90 \%$ reaching the source, but only $24 \%$ flying upwind to a 35:65 Z/E ratio, and none to the 50:50 blend. Although BE males also displayed a preference for the identified 1:99 Z/E11-14:OAc blend, they also reached the source in approximately equivalent proportions with a $65: 35 \mathrm{Z} / \mathrm{E} 11$ 14 :OAc mix, and $12 \%$ of the tested males exhibited complete flights to a source, releasing a 35:65 Z/E11-14:OAc mix. The combined results from experiment 2 and previous studies of ECB suggest that with respect to specificity for odor quantity and quality, the three populations can be ranked: $\mathrm{UZ}>\mathrm{BE}>\mathrm{ACB}$.

Experiment 3: Are There Rare ACB Males that Respond to the ECB Blends?

Rare males were found in the ACB population that exhibited complete flights to the UZ ECB pheromone source (30 $\mu$ g, Fig. 3). Of 525 ACB males tested to the UZ blend, 3\%

Fig. 3 Percentage source contact of male UZ ECB and male ACB to the $30-\mu \mathrm{g}$ source of the UZ (97:3 Z/E11-14:OAc) and 30- $\mu \mathrm{g}$ source of the ACB (2:1 Z/E12-14: OAc) pheromone blends. For UZ ECB, 256 males were tested to the ACB blend, with $4 \%(N=10)$ reaching the source. Of the UZ males that did not fly upwind to the ACB blend 213 were then tested to the UZ blend, with $97 \%$ $(N=207)$ reaching the source. For ACB, 525 males were tested to the UZ blend, with $3 \%(N=16)$ reaching the source. Of the ACB males that did not fly upwind to the UZ blend 250 were then tested to the ACB blend, with $56 \%(N=140)$ reaching the source

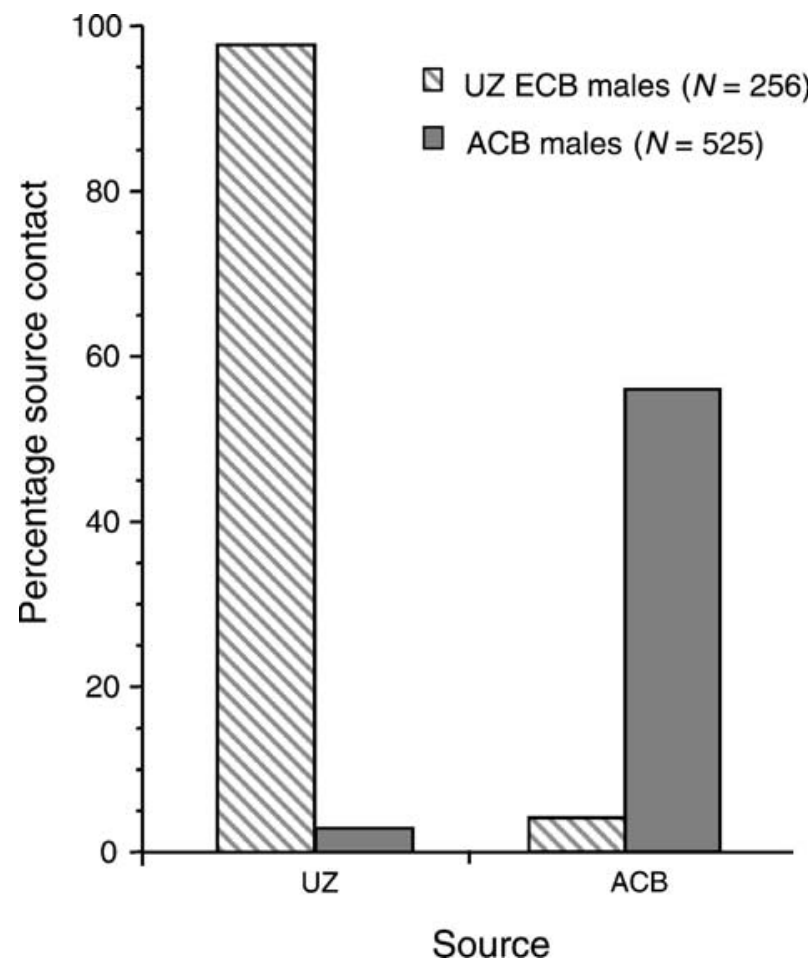


(16 males) made source contact. These same 16 males also flew upwind to the $30-\mu \mathrm{g}$ dose of the ACB blend. Additionally, of the 509 ACB males tested to the UZ blend that did not fly upwind to that source, 250 were also tested to the ACB blend, with $56 \%(N=140)$ making source contact.

For UZ males, 256 were tested to the $30-\mu \mathrm{g}$ dose of the ACB blend, with $4 \%$ (10 males) reaching the source (Fig. 3). These same 10 males also flew upwind to the $30-\mu \mathrm{g}$ dose of the UZ blend. Additionally, of the UZ males tested to the ACB blend that did not fly upwind to that source, 213 were also tested to the UZ blend, with $97 \%(N=207)$ making source contact.

In a second testing protocol to detect the presence of rare ECB blend-responding ACB males, ACB males were tested to $30-\mu \mathrm{g}$ doses of the $\mathrm{UZ}$ and BE blends, and to $30 \mu \mathrm{g}$ of the ACB blend. Of 460 males tested, 61\% $(N=281)$ made contact with the ACB source; $4 \%$ $(N=18)$ reached the $\mathrm{UZ}$ and ACB sources; 3\% $(N=14)$ reached the BE and ACB sources; and $1 \%(N=5)$ flew upwind to all three sources (Fig. 4).

Experiment 4: Effect of Z9-14:OAc on Male ACB Response to the ACB Pheromone Blend

The percentage of ACB males flying upwind to the $30-\mu \mathrm{g}$ dose of the ACB blend was reduced when 1\% Z9-14:OAc was added to the ACB blend (Fig. 5; Fisher's exact test $P<$ $0.001, d f=1)$. With the ACB blend alone, $66 \%(N=129)$ of the males reached the source, but only $30 \%$ did so when $1 \%$ Z9-14:OAc was added. In comparison, $77 \%$ of ECB males from the BE colony reached the source in response to the BE blend $(30 \mu \mathrm{g})$ alone, but only

Fig. 4 Percentage source contact by male $\mathrm{ACB}(N=460)$ tested in sequence to the BE (1:99 Z/E1114:OAc; $30 \mu \mathrm{g})$, UZ (97:3 Z/E1114:OAc; $30 \mu \mathrm{g})$, and ACB (2:1 Z/ E12-14:OAc; $30 \mu \mathrm{g}$ ) pheromone blends. Response values show percentage of males reaching the source of the ACB blend alone $(61 \%, N=281)$, those reaching the ACB and UZ sources $(4 \%$, $N=18)$; those reaching the $\mathrm{BE}$ and ACB sources $(3 \%, N=14)$; and those flying upwind to all three blends $(1 \%, N=5)$. None of the ACB males flew upwind to one or both of the ECB blends, but not to $\mathrm{ACB}$ blend

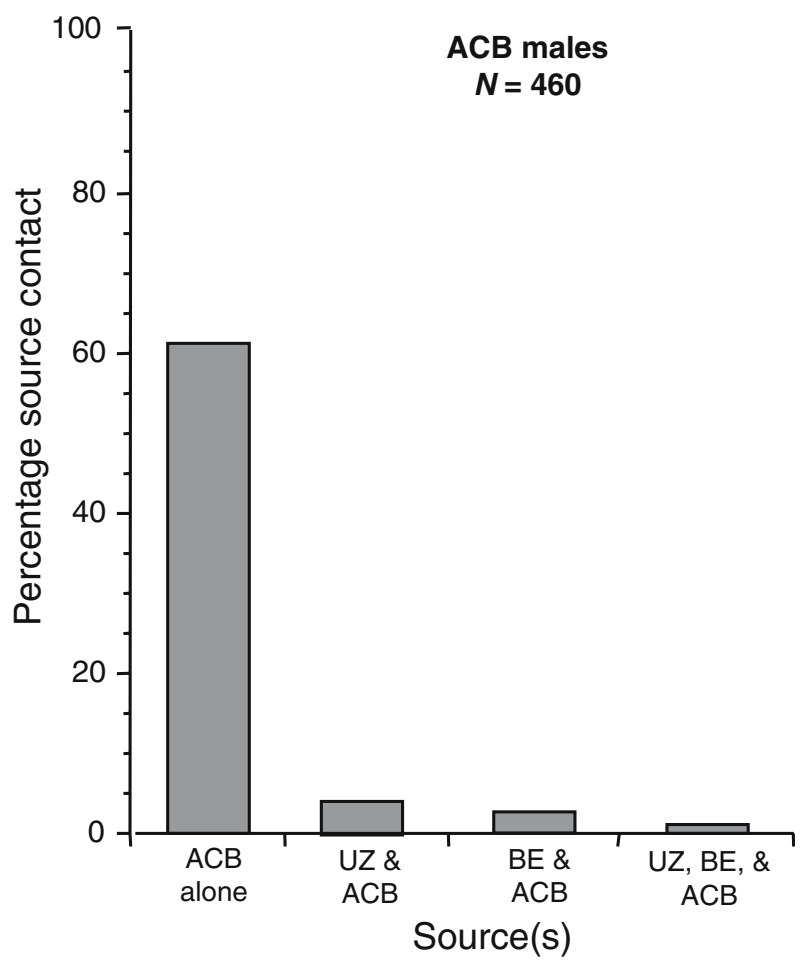



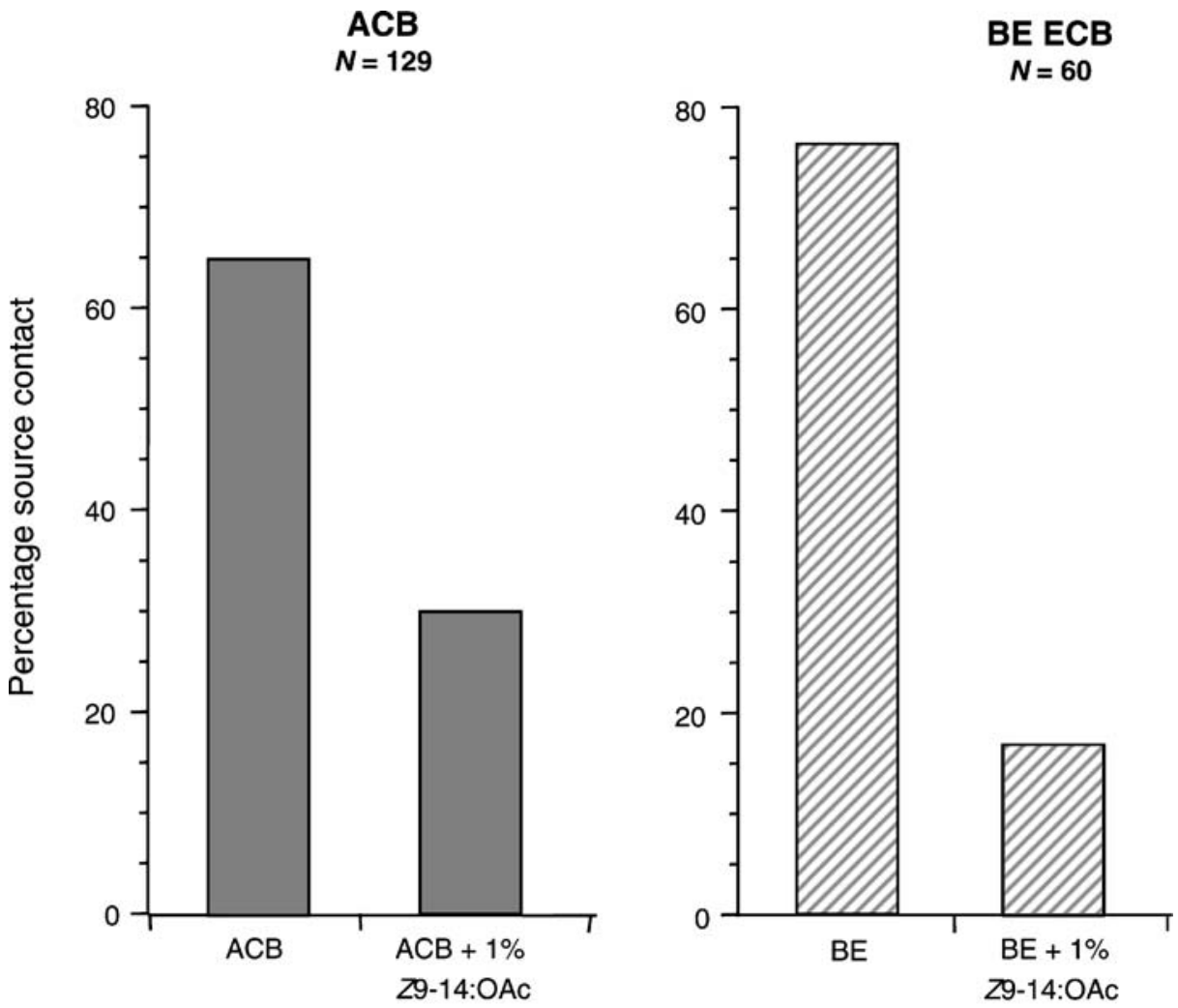

Source

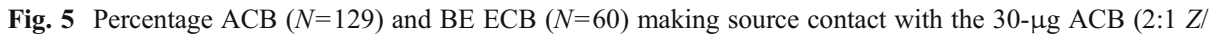
E12-14:OAc) or the BE (1:99 Z/E11-14:OAc) blend and then to the ACB blend with 1\% Z9-14:OAc added, or the BE blend with $1 \%$ Z9-14:OAc added

$17 \%$ did so when $1 \%$ Z9-14:OAc was added to the blend (Fig. 5; Fisher's exact test $P<$ $0.001, d f=1)$.

Experiment 5: Response of ACB Males to the Combined ACB/ECB Blends

The upwind flight response of ACB males to a 30- $\mu \mathrm{g}$ dose of the ACB blend was unaffected by the addition of the $\mathrm{UZ}$ or BE blend $(30 \mu \mathrm{g})$. With the ACB blend alone, $66 \%$ of the tested males $(N=64)$ reached the source, and when the UZ or BE blend was added $68 \%(N=60$; Fisher's exact test $P=0.894, d f=1)$ and $57 \%(N=65$; Fisher's exact test $P=$ $0.368, d f=1)$ of ACB males, respectively, reached the source.

Experiment 6: Response of ACB Males to the ACB Blend with 14:OAc Added

The upwind flight response of ACB males to the ACB blend also was unaffected by the addition of 14:OAc. With the 30- $\mu$ g dose of the ACB blend alone, $64 \%$ of the tested males 
$(N=55)$ reached the source, and when 50\% 14:OAc was added to the ACB blend, $60 \%(N=$ 50; Fisher's exact test $P=1.000, d f=1$ ) reached the source.

\section{Discussion}

Our results provide several important findings concerning the response specificity of ACB males for their pheromone, and allow comparisons with previously published results for the closely related ECB. First, there are rare ACB males that respond to their own pheromone and to one or both of the ECB blends. Second, the upwind flight response of ACB males was antagonized by the addition of Z9-14:OAc to the pheromone blend, similar to ECB, but was unaffected by addition of either of the ECB blends. Third, ACB males responded in high proportions to a wider range of blend ratios than did ECB males. Fourth, addition of 14:OAc, a previously identified compound in ACB pheromone glands, did not affect the upwind flight response of ACB males to the 2:1 Z/E12-14:OAc blend.

\section{Rare ACB Males Responding to the ECB Pheromone Blends}

Male ACB were found that responded to the ECB UZ blend (4\%), the BE blend (3\%), and to both the $\mathrm{UZ}$ and $\mathrm{BE}$ blends (1\%), in addition to the ACB blend. The upwind flight percentages are comparable with levels of $3-5 \%$ for ECB males of the UZ and BE races that responded to the ACB blend (Linn et al., 2003). The finding that some ACB males can detect and respond to the ECB pheromone blends adds to a growing list of cases involving the presence of broadly responsive individuals in moth populations (Baker, 2002) that have the capacity to process information about pheromone components utilized by related species in an agonistic manner. The adaptive value of being a broad responder is that these individuals have the potential to exploit new communication channels, as has been proposed for the ACB (Baker, 2002; Roelofs et al., 2002). The finding also is not entirely unexpected given the proposed phylogenetic relationships in the Ostrinia (Ishikawa et al., 1999a, b). Most of the Ostrinia species in Asia, from which the ACB were derived (Roelofs et al., 2002), utilize Z/E11-14:OAc mixtures (Ishikawa et al., 1999a) and, thus, there presumably exists the genetic capacity in ACB to express the ECB receptors. The finding of rare males in both ECB and ACB also affords the opportunity to explore the peripheral processing of these compounds in rare and normal individuals in both species (Domingue et al., 2006).

Functional Role of Z9-14:OAc and the Evolution of Pheromone Communication in Ostrinia

Similar to ECB, the upwind flight response of significant numbers of male ACB was antagonized by the addition of Z9-14:OAc to the ACB blend. Glover et al. (1989) showed that addition of $1 \%$ Z9-14:OAc reduced the level of upwind flight by BE ECB males from $73 \%$ to $18 \%$, which is similar to the values reported here for $\mathrm{BE}$ males $(77 \%$ reduced to $17 \%$, Fig. 5). The addition of $1 \%$ Z9-14:OAc to the ACB blend also resulted in a reduction of upwind flight levels by ACB from $66 \%$ to $30 \%$.

The observed antagonist effect of Z9-14:OAc on ACB upwind flight is of interest because of its impact on discussions on the phylogenetic relationships within Ostrinia, as proposed by Ishikawa et al. (1999a, b). In a study of the host plants and sex pheromones of 
Ostrinia species in Japan, Ishikawa et al. (1999a) showed that all species use the $\Delta 11$ pheromone components, similar to those of ECB, except for the ACB populations, which use the $\Delta 12$ pheromone components. It was proposed that the ACB populations are derived from one of the closely related Ostrinia populations that use the $\Delta 11$ components. Support for this hypothesis comes from a study of the reductases of ACB. Evolution of the unusual pheromone components of $\mathrm{ACB}$ should require a change in the desaturase and a change in the specificity of the reductase for $\Delta 12$ instead of $\Delta 11$ compounds. However, a study of the ACB reductases (Zhao et al., 1995) revealed that their $\Delta 12$ reductase was able to convert Z11-14:Acid to the corresponding alcohol more efficiently than it did for the $\Delta 12-14$ :Acids (with which it seemed to favor the $E$ isomer). This suggests a possible close linkage to a Z11 strain of Ostrinia.

In the study by Ishikawa et al. (1999a), two phylogenies were proposed. In the first, based on morphological data (Mutuura and Munroe, 1970), the group III trilobed uncus species group is divided into two subgroups: one containing ACB, ECB, and Ostrinia orientalis; and a second containing Ostrinia scapulalis, Ostrinia zaguliaevi, and Ostrinia zealis. In the second proposed phylogeny, based on mitochondrial COII gene sequence analysis (Ishikawa et al., 1999b; Kim et al., 1999), the subgroups of group III are not supported, and ACB is placed basal to the remaining five species. Based on the existing knowledge about the pheromone systems in Ostrinia, the following sequence of changes were proposed to have occurred: (1) the most ancestral form (as yet unidentified) most likely utilized E11-14:OAc alone, based on the fact that one of the most basal species, Ostrinia latipennis, uses only E11-14:OH; (2) species evolved that used mixtures of Z/E1114:OAc, such as Ostrinia palustralis; (3) species evolved using Z/E11-14:OAc + Z9-14: $\mathrm{OAc}$ as a three-component blend (O. zaguliaevi and $O$. zealis); (4) species evolved, such as ECB and O. scapulalis, in which Z9-14:OAc has switched functionality and is an antagonist component of the communication system. Within this scheme, the ACB is hypothesized to be ancestral to the group that includes $O$. zaguliaevi and O. zealis, that is, before the incorporation of Z9-14:OAc into the pheromone as an agonist component, and in agreement with the second phylogeny based on COII gene sequence analysis. However, if this scheme is correct, then our result, showing that Z9-14:OAc also is an antagonist of upwind flight in $\mathrm{ACB}$, implies that the role of Z9-14:OAc arose earlier than previously thought, and further that the functionality of this component has switched more than once. Alternatively, our result suggests that species using the three-component pheromone constitute a group that is ancestral to ACB, ECB, and O. scapulalis in which Z9-14:OAc is an antagonist. This hypothesis is more in agreement with the phylogenetic relationships based on morphological data (Mutuura and Munroe, 1970; Ishikawa et al., 1999a), and the idea that agonist functionality precedes the emergence of antagonism in the evolution of pheromone systems (Linn and Roelofs, 1995; Baker, 2002).

Finally, the addition of the ECB blends did not affect the upwind flight of ACB males to the ACB blend. This result supports the hypothesis that the lack of response of ACB males to the ECB pheromone in experiment 3 was not because of antagonistic properties of the ECB pheromone components, but rather threshold differences between rare ECBresponding $\mathrm{ACB}$ males compared with nonresponding $\mathrm{ACB}$ males to the $\mathrm{ECB}$ blends.

\section{Response Specificity and Additional Pheromone Components for ACB}

Male ACB displayed a relatively low level of specificity with respect to blend quality, compared with ECB, evidenced by the similar levels of upwind flight and source contact 
( $45 \%$ to $66 \%)$ that occurred with a range of ratios (4:1 to $1: 4)$ of $Z / E 12-14: \mathrm{OAc}$, and the variability in response over the course of the experiments. Previous studies with ECB (Figs. 1b and 2b; Linn et al., 1997) showed that UZ males exhibited a greater specificity for blend ratio than did BE males, with no UZ males flying upwind to a 50:50 ratio. BE males responded to a wider range of ratios (1:99 to 35:65 Z/E11-14:OAc). With respect to odor quantity, lower specificity was also evidenced by the fact that the maximal level of upwind flights by ACB males was $66 \%$, a value that is considerably less than routinely observed with the UZ ECB males ( $>90 \%$; see Linn et al., 1997, 2003), but comparable to maximal levels observed with the BE ECB race to their 1:99 Z/E11-14:OAc mixture (65\% to 70\%).

Results of other studies with ACB suggest that throughout Asia a range of Z/E12-14: OAc mixtures are produced by females and result in trap captures of males (Ando et al., 1980; Klun et al., 1980; Cheng et al., 1981; Yeh et al., 1989; Boo and Park, 1998). Field trapping studies and analysis of female pheromone glands at sites in Japan led Huang et al. (2002) to hypothesize that there was a pheromone polymorphism, with populations in the north preferring a blend containing $36 \%$ to $39 \%$ E12-14:OAc, and in the south $44 \% E 12$ 14:OAc. Analysis of field trapping studies in South Korea showed that high numbers of moths were captured with ratios from 3:1 to 1:3 E/Z12-14:OAc (Boo and Park, 1998).

One explanation for the relatively lower response levels and lack of specificity in ACB is that because the pheromone is made up of unusual $\Delta 14$ compounds, there is less pressure from interspecies encounters that could lead to mating mistakes, as might occur in other Ostrinia that utilize $\Delta 11$ compounds. It also could be the case that other pheromone components are involved in the communication system. Boo and Park (1998) reported the presence of 14:OAc in hexane extracts of female abdominal tips from 2- to 3-d-old females that contained a mean ratio of $0.74: 1: 1.36$ for $14: \mathrm{OAc}, E 12$ and Z12-14:OAc, but the compound did not elicit an EAG response and was not used in field trials. The field trials showed that among 12 blends tested, the greatest capture occurred with the 2:1 Z/E mix, but as noted above there was significant variability among sites and in the range of blends capturing high numbers of moths. At one site, a ginger field, maximal captures were reported with a 5:1 E/Z mixture. Kou et al. (1992) also reported the presence of 14:OAc in the pheromone gland analysis of moths in Taiwan. However, in field trials there was no difference between the three- and two-component mixtures, or a 3:2 Z/E12 formulation identified in Japan (Ando et al., 1980). Finally, 14:OAc was reported in pheromone gland tips of females in China (Cheng et al., 1981), but when added to the 1:1 Z/E12 mix at the reported gland content $(1.8 \times$ sum of isomers), it resulted in reduced trap capture. Our results also suggest that lower levels of upwind flight were not because of a lack of 14:OAc as a potential pheromone component.

Acknowledgements We thank Kathy Poole for help in maintaining the ACB and ECB colonies, and Satoshi Nojima and Shannon Olsson for preparing the solutions for flight tunnel tests. The project was funded by NSF IBN \#034340.

\section{References}

Ando, T., Saito, O., Arai, K., and TAKahashi, N. 1980. (Z)- and (E)-12-Tetradecenyl acetates: sex pheromone components of oriental corn borer (Lepidoptera: Pyralidae). Agric. Biol. Chem. 44:26432649 . 
BAKER, T. C. 2002. Mechanism for saltational shifts in pheromone communication systems. Proc. Natl. Acad. Sci. USA 99:13368-13370.

Boo, K. S. and PARK, J. W. 1998. Sex pheromone of the Asian corn borer moth, Ostrinia furnacalis (Guenée) (Lepidoptera: Pyralidae) in South Korea. J. Asia Pacific Entomol. 1:77-84.

Cheng, Z. Q., XiaO, J. C., Huang, X. T., Chen, D. L., Li, Q. J., Li, Y. S., Huang, S. R., LuO, Q. C., Yang, C. M., and YANG, T. H. 1981. Sex pheromone components isolated from China corn borer, Ostrinia furnacalis, Guenée (Lepidoptera: Pyralidae), (E)- and (Z)-12-tetradecenyl acetates. J. Chem. Ecol. 7:841-851.

Cossé, A. A., Campbell, M. G., Glover, T. J., Linn, C. E. Jr., Todd, J. L., Baker, T. C., and Roelofs, W. L. 1995. Pheromone behavioral responses in unusual male European corn borer hybrid progeny not correlated to electrophysiological phenotypes of their pheromone-specific antennal neurons. Experientia 51:809-816.

Domingue, M. J., Roelofs, W. L., Linn, C. E. JR., and BaKer, T. C. 2006. Effects of egg-to-adult development time and age on olfactory neuron response to semiochemicals in European corn borers. $J$. Insect Physiol. 52:975-983.

Du, J. W., ZHU, T. P., ZHANG, T. P., XU, S. F., and DAI, X. J. 1986. Studies on the precise blending of the pheromone components of the Asian corn borer, Ostrinia furnacalis Guenée (Lep. Pyralidae). Contrib. Shanghai Inst. Entomol. 6:17-22.

Glover, T. J., Perez, N., and Roelofs, W. L. 1989. Comparative analysis of sex-pheromone-response antagonists in three races of European corn borer. J. Chem. Ecol. 15:863-873.

Huang, Y., Takanashi, T., Hoshizaki, S., Tatsuki, S., Honda, H., Yoshiyasu, Y., and Ishikawa, Y. 1998. Geographic variation in the sex pheromone of Asian corn borer, Ostrinia furnacalis, in Japan. $J$. Chem. Ecol. 24:2079-2088.

Huang, Y., Takanashi, T., Hoshizaki, S., Tatsuki, S., and Ishikawa, Y. 2002. Female sex pheromone polymorphism in adzuki bean borer, Ostrinia scapulalis, is similar to that in European corn borer, $O$. nubilalis. J. Chem. Ecol. 28:533-539.

Ishikawa, Y., Takanashi, T., Kim, C.-G., Hoshizaki, S., TATsuki, S., and HuAng, Y. 1999a. Ostrinia spp. in Japan: their host plants and sex pheromones. Entomol. Exp. Appl. 91:237-244.

IshiKaWA, Y., TAKANASHI, T., and HuANG, Y. 1999b. Comparative studies on the sex pheromones of Ostrinia spp. in Japan: the burdock borer, Ostrinia zealis. Chemoecology 9:25-32.

Kim, C., Hoshizaki, S., HuAng, Y., TATsuki, S., and Ishikawa, Y. 1999. Usefulness of mitochondrial COII gene sequences in examining phylogenetic relationships in the Asian corn borer, Ostrinia furnacalis, and allied species (Lepidoptera: Pyralidae). Appl. Entomol. Zool. 34:405-412.

Klun, J. A., Bierl-Leonhardt, B. A., Schwarz, M., Litsinger, J. A. Barrion, A. T., Chiang, H. C., and JIANG, Z. 1980. Sex pheromone of the Asian corn borer moth. Life Sci. 27:1603-1606.

Kochansky, J., Cardé R. T., Liebherr, J., and Roelofs, W. L. 1975. Sex pheromones of the European corn borer in New York. J. Chem. Ecol. 1:225-231.

Kou, R., Ho, H. Y., YAnG, H. T., ChOw, Y. S., and WU, H. J. 1992. Investigation of sex pheromone components of female Asian corn borer, Ostrinia furnacalis (Hübner) (Lepidoptera: Pyralidae) in Taiwan. J. Chem. Ecol. 18:833-840.

LinN, C. E. JR. and Roelofs, W. L. 1995. Pheromone communication in the moths and its role in the speciation process, pp. 263-300, in D. Lambert and H. Spencer (eds.). Speciation and the Recognition Concept: Theory and Application. Johns Hopkins University Press, Baltimore, MD.

Linn, C. E. JR., Young, M. S., Gendle, M., Glover, T. J., and RoelofS W. L. 1997. Sex pheromone blend discrimination in two races and hybrids of the European corn borer moth, Ostrinia nubilalis. Physiol. Entomol. 22:212-223.

LinN, C. E. JR., O'CONNOR, M., and Roelofs, W. L. 2003. Silent genes and rare males: a fresh look at pheromone blend response specificity in the European corn borer moth, Ostrinia nubilalis. J. Insect Sci. 3:15 (Available online: http://www.insectscience.org/3.15).

Löfstedt, C., Hansson, B. S., Roelofs, W. L., and Bengtsson, B. O. 1989. No linkage between genes controlling female pheromone production and male pheromone response in the European corn borer Ostrinia nubilalis Hübner (Lepidoptera: Pyralidae). Genetics 123:553-556.

MutuUrA, A. and Munroe, E. 1970. Taxonomy and distribution of the European corn borer and allied species: genus Ostrinia (Lepidoptera: Pyralidae). Mem. Entomol. Soc. Can. 71:1-112.

Roelofs, W. L., Du, J. W., TAnG, X. H., Robbins, P. S., and Eckenrode, C. J. 1985. Three European corn borer populations in New York based on sex pheromones and voltinism. J. Chem. Ecol. 11:829-836.

Roelofs, W. L., Glover, T. J., TAng, X.-H., Sreng, I., Robbins, P., Eckenrode, C. J., Löfstedt, C., Hansson, B. S., and Bengtsson, B. O. 1987. Sex pheromone production and perception in European corn borer moths requires both autosomal and sex-linked genes. Proc. Natl. Acad. Sci. USA 84:75857589 . 
Roelofs, W. L., LiU, W., HaO, G., JiaO, H., Rooney, A. P., and LinN, C. E. JR. 2002. Evolution of moth sex pheromones via ancestral genes. Proc. Natl. Acad. Sci. USA 99:13621-13626.

Yeh, S., LeE, K., Chang, K.-T., Yen, F.-C., and Hwang, J. S. 1989. Sex pheromone components from Asian corn borer, Ostrinia furnacalis (Lepidoptera: Pyralidae) in Taiwan. J. Chem. Ecol. 15:497-505.

ZHAO, C.-H., LU, F., BENGTSSON, M., and LÖFSTEDT, C. 1995. Substrate specificity of acetyltransferase and reductase enzyme systems used in pheromone biosynthesis by Asian corn borer, Ostrinia furnacalis. J. Chem. Ecol. 21:1495-1509. 